\title{
Multimotors Coordination Control Method for Consistent Total Traction Torque of Electric Locomotives
}

\author{
Jianhua Liu, ${ }^{1}$ Rui Nie, ${ }^{1}$ and Zheshu Li (iD ${ }^{2}$ \\ ${ }^{1}$ College of Traffic Engineering, Hunan University of Technology, Zhuzhou 412007, China \\ ${ }^{2}$ College of Music, Hunan University of Technology, Zhuzhou 412007, China \\ Correspondence should be addressed to Zheshu Li; zheshuli0605@163.com
}

Received 18 January 2020; Revised 4 August 2020; Accepted 18 August 2020; Published 1 September 2020

Academic Editor: Ziyou Gao

Copyright (c) 2020 Jianhua Liu et al. This is an open access article distributed under the Creative Commons Attribution License, which permits unrestricted use, distribution, and reproduction in any medium, provided the original work is properly cited.

Multimotors traction is a common driving mode for electric locomotives. The complexity and variability of the operating environment often cause difficulties for one or some traction motors to output the distributed traction torque, which requires the redistribution of the torque output of multimotors to maintain the total traction torque of all motors at a given constant. To this end, a multimotors coordination control method was proposed for consistent total traction torque of electric locomotives. Through this approach, a traction torque-coordinated control structure was constructed on the basis of energy minimisation with an optimal dynamic adjustment mode. The sliding mode tracking control was developed for each independent motor to improve its dynamic response performance. A simulation verification of motors fault types in two different cases was conducted. The results showed that the proposed method could track the total traction torque in a short time, and the system fluctuations in independent motor coordination process were very small.

\section{Introduction}

Most of the existing electric locomotives use multiple motors together to provide traction torque [1]. For example, the traction power of the $\mathrm{SS}_{8}$-type electric locomotive is provided by four motors together. When the locomotive is under traction condition, the microcomputer control system calculates the total traction torque required according to the driver's handling instruction and then distributes it to multimotors mounted on the bogie according to the present coordination strategy [2-5].

The locomotive can be safely and smoothly operated only when the sum of all the traction torque provided by multimotors is consistent with the total traction torque required [6]. However, the complexity and variability of the operating environment often cause one or some traction motors to lose traction performance. For example, when the locomotive suddenly enters the rail covered with ice and snow from the normal rail surface, some power wheels often lose traction due to slippage [7-9], resulting in the decrease of the total traction torque. Especially for locomotive on uphill slopes, the backward failure may occur if the required total traction torque cannot be maintained [10]. This phenomenon needs to solve two problems: one is to coordinate the output traction torque among multimotors ensuring that the vector sum of all the traction torque provided by multimotors is consistent with the total traction torque required, and another is to achieve the optimal dynamic performance for independent motors.

In the field of multimotors coordination control methods, scholars at home and abroad have conducted considerable research work regarding decentralised coordination, adaptive robust, and circular cross-coupling control methods [11-13]. In [14], the multimotors coordination control system was considered as an integrated system with multiple dynamic interval subsystems, and a decentralised coordination control strategy was established to solve the system uncertainty. In [15], a coordinated selfadaptive robust control method was proposed to weaken the influence of load variation and system uncertainty. In [16], a consistency algorithm was introduced into the multiaxis synchronous control system to achieve the high- 
performance synchronous control of the multimotors system. In [17], a multimotors synchronous process coupling control system was designed to improve the synchronisation performance by changing the synchronisation and tracking coefficients in real time in speed feedback and synchronisation compensation. However, according to the latest information mastered by the author, the existing multimotors coordination control method is mostly used to ensure the coordination of a certain state variable, such as position or/and speed. For the problem of consistent total traction torque, although the author and his colleges have made an innovative attempt, many significant scientific problems must be solved because a detailed allocation algorithm of the torque output of the traction system is unavailable in [18].

The dynamic response performance of an independent motor is directly related to the overall performance of the multimotors coordination process. In [19], a suitable quasisliding mode observer with low sampling frequency was designed to estimate the rotor position and stator flux; then the tracking control of the maximum power point of wind turbine was realised. In [20], a fault reconstruction method based on sliding mode variable structure was proposed. In [21], the direct torque and flux control without the beat was introduced into the motor control, which realised the minimisation control of rapid torque and dynamic loss of the motor. In [22], a feedback linearization direct torque control method based on space vector modulation was proposed to reduce the influence of electromagnetic torque and stator flux pulsation.

For electric locomotives, the traction torque of all motors is in the same direction; when one or some motors lose traction performance, there are an infinite number of adjustment methods to coordinate the torque output of the remaining motors, such that the total traction torque is constant. But the optimal dynamic adjustment procedure is minimizing the energy function composed of the traction torques [23-25]. For this purpose, the $\mathrm{SS}_{8}$-type electric locomotive with four motors that provide traction torque was taken as the research object in this work; a multimotors coordination control framework with consistent total traction torque was constructed. A multimotors coordination control model based on energy minimisation was proposed to ensure the minimum energy of the multimotors dynamic adjustment process through the optimisation of the weight matrix. Meanwhile, the traction torque control based on sliding mode variable structure was constructed to improve the dynamic response performance of each independent motor.

The structure of this paper is as follows. In Section 2, the $\mathrm{SS}_{8}$-type electric locomotive is taken as the research object, and a multimotor coordination control strategy for consistent total traction torque is proposed. Section 3 elucidates the traction torque output coordination method based on energy minimisation. Section 4 designs the sliding mode variable structure controller for each independent motor. Section 5 includes the experimental and data analysis and Section 6 is the conclusion.

\section{Multimotors Coordination Control Strategy}

The $\mathrm{SS}_{8}$-type electric locomotive is an $\mathrm{AC} / \mathrm{DC}$ transmission phase-controlled electric locomotive [26]. The locomotive adopts B0-B0 axis structure, which belongs to the four-axis passenger locomotive, and uses AC-DC traction mode, with each motor equipped with four traction motors and transmits traction torque through a flat pull rod [27]. When the locomotive is running, the locomotive traction control initially calculates the required total traction power after receiving the driver's handle command and then assigns it to the four motors mounted on the bogie according to a predetermined strategy. In fact, the effect of output traction torque on drive shaft is to provide longitudinal force; this means that the output traction torque of each motor acts in the same direction, so the basic condition for the safe and stable operation of electric locomotives indicates that the sum of all the traction torque provided by all motors should be consistent with the total traction torque required for the locomotive to operate; that is,

$$
\sum_{j=1}^{4} T_{e j}=T_{e}^{*},
$$

where $T_{e j}$ is the output traction torque of motor $j$ and $T_{e}^{*}$ is the required total traction torque according to the handle command.

So, a multimotors coordination control strategy is constructed to ensure that the total traction torque is consistent when one or some motors lose traction torque output; meanwhile, the dynamic response performance of each independent motor is improved (see Figure 1).

Figure 1 shows that the coordination controller integrates the traction torque output of each motor to compare it with the given total traction torque required. Based on the principle of minimized energy function, the real-time traction torque output state of each motor is redistributed to ensure that the sum of the traction torque of each motor $\sum_{j=1}^{4} T_{e j}$ is consistent with the total traction torque $T_{e}^{*}$. At the same time, a sliding mode controller (SMC) is proposed to ensure that each direct-current motor (DCM) has preferable dynamic response performance.

The mathematical model of permanent magnet brushless DCM can be expressed as [28]

$$
\left\{\begin{array}{l}
L_{j} \frac{\mathrm{d} i_{j}}{\mathrm{~d} t}=-R_{j} i_{j}-k_{e j} k_{t j} \omega_{j}+u_{j}, \\
\left(J_{1 j}+k_{t j}^{2} J_{0 j}\right) \dot{\omega}_{j}=-\left(b_{1 j}+k_{t j}^{2} b_{0 j}\right) \omega_{j}+T_{e_{j}}-T_{L_{j}}, \\
T_{e_{j}}=k_{t j} k_{m j} i_{j},
\end{array}\right.
$$

where $R_{j}$ and $L_{j}$ represent the resistance and inductance of the armature circuit, respectively; $i_{j}$ is the motor current; $\omega_{j}$ is the output angular velocity of motor gearbox $j ; u_{j}$ is the voltage of the input port of the armature circuit; $k_{e j}$ is the back electromotive force constant; $k_{t j}$ is the gear ratio of the gearbox; $J_{0 j}$ and $J_{1 j}$ are the moments of inertia of the motor 


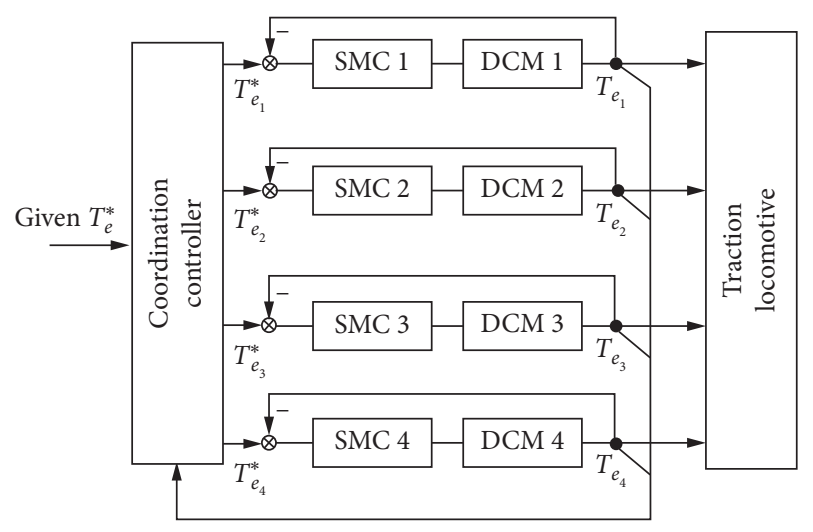

FIGURE 1: Multimotors coordination control strategy for consistent total traction torque.

and gearbox; $b_{0 j}$ and $b_{1 j}$ are the viscous friction coefficients of the motor and the gearbox; $k_{m j}$ is the motor torque constant; $T_{L j}$ is the load torque; and $T_{e j}$ is the output torque of the motor.

Set $x_{1 j}=\omega_{j}, x_{2 j}=\dot{\omega}_{j}$, and $x_{3 j}=T_{e j}$; the state equation of (2) can be expressed as

$$
\left\{\begin{array}{l}
\dot{x}_{1 j}=-\frac{b_{e q j}}{J_{e q j}} x_{1 j}+\frac{x_{3 j}}{J_{e q j}}+d_{1 j}, \\
\dot{x}_{2 j}=-a_{0 j} x_{1 j}-a_{1 j} x_{2 j}+b_{j} u_{j}+d_{2 j}, \\
\dot{x}_{3 j}=-J_{e q j} a_{0 j} x_{1 j}+\left(b_{e q j}-J_{e q j} a_{1 j}\right) x_{2 j}+J_{e q j} b_{j} u_{j}+d_{3 j},
\end{array}\right.
$$

where $J_{e q j}=J_{1 j}+k_{t j}^{2} J_{0 j}$ is the equivalent moment of inertia; $b_{e q j}=b_{1 j}+k_{t j}^{2} b_{0 j}$ is the equivalent viscous damping constant; and $b_{j}=\left(k_{t j} k_{m j}\right) / J_{e q j} L_{j}, a_{0 j}=\left(k_{t j}^{2} k_{m j} k_{e j}+R_{j} b_{e q j}\right)$ $/ J_{\text {eqj }} L_{j}$, and $a_{1 j}=\left(b_{\text {eqj }} / J_{\text {eqj }}\right)+R_{j} / L_{j}$; besides, $d_{j}=\left[d_{1 j}, d_{2 j}\right.$, $\left.d_{3 j}\right]=\left[-\left(T_{L j} / J_{e q j}\right),-\left(R_{j} / J_{e q j} L_{j}\right) T_{L j}-\left(1 / J_{e q j}\right) T_{L j},-\left(R_{j} / L_{j}\right)\right.$ $\left.T_{L j}\right]$ are the time-varying disturbances.

The load torque of the motor cannot be infinitely large; that is, $\left|T_{L j}\right| \leq \beta_{j}$. The axis end of the motor is mechanically connected to the wheel-set through the gearbox. Moreover, the load torque can be considered slow, time-varying, or substantially constant; that is, $\left|\dot{T}_{L j}\right| \leq \lambda_{j}$. Therefore, the timevarying disturbance $d_{j}$ is bounded; that is, $\left\|d_{j}(t)\right\| \leq \xi_{i}$, where $\beta_{j}, \lambda_{j}, \xi_{i}$ are all positive constants.

\section{Multimotors Coordination Based on Energy Minimization}

The multimotors coordination procedure is the dynamic change procedure of multimotors output traction torque, in which the energy function is composed of each traction torque reaching the minimum, which indicates the optimal dynamic adjustment process [29]. The energy function of traction torque can be expressed as

$$
\begin{aligned}
& J=\frac{1}{2} T^{T} P T=\frac{1}{2}\left[T_{e 1}, T_{e 2}, T_{e 3}, T_{e 4}\right]\left[\begin{array}{llll}
a_{1} & & & \\
& a_{2} & & \\
& & a_{3} & \\
& & & a_{4}
\end{array}\right]\left[\begin{array}{c}
T_{e 1} \\
T_{e 2} \\
T_{e 3} \\
T_{e 4}
\end{array}\right] \\
& =\frac{1}{2} a_{1} T_{e 1}^{2}+\frac{1}{2} a_{2} T_{e 2}^{2}+\frac{1}{2} a_{3} T_{e 3}^{2}+\frac{1}{2} a_{4} T_{e 4}^{2},
\end{aligned}
$$

where $J$ is the energy function that is composed of each traction torque; $T$ is the traction torque matrix; and $P$ is the weight matrix, and $P \in R_{4 \times 4}$. The main diagonal element corresponds to the weight coefficient $a_{j}$ of each torque, and $a_{j}>0$.

For the electric locomotive traction system that multiple motors provide power together, due to the different axle weights of the driving wheels, the traction torque provided by corresponding motor may have different effects on traction performance. The purpose of using the weight coefficient $a_{j}$ is to reduce the traction fluctuation amplitude of motor that will cause greater degree of influence and, at the same time, to increase the traction adjustment amplitude of motor with a smaller degree of influence. So, the multimotors traction torque coordination method based on energy minimisation can be expressed as a constrained optimisation problem; that is,

$$
\begin{aligned}
& \min J=\min \sum_{j=1}^{4} \frac{1}{2} a_{j} T_{e j}^{2} \\
& \text { s.t. }\left\{\begin{array}{l}
\sum_{j=1}^{4} T_{e j}=T_{e}^{*}, \\
0 \leq T_{e j} \leq b,
\end{array}\right.
\end{aligned}
$$

where $b$ is a positive constant.

Formula (5) can be solved by using the Karush-Kuhn-Tucker (KKT) conditions. The equality constraint of (5) can be expressed as $h\left(T_{e j}\right)=\sum_{j=1}^{4} T_{e j}-T_{e}^{*}=0$; the inequality constraints of it can be expressed as $g_{1 j}\left(T_{e j}\right)=$ $0-T_{e j} \leq 0$ and $g_{2 j}\left(T_{e j}\right)=T_{e j}-b \leq 0$, respectively. Moreover, the inequality constraint of (5) can be transferred to equality constraint by introducing slack variables,

$$
\begin{aligned}
& h_{1 j}\left(T_{e j}, a_{1 j}\right)=g_{1 j}\left(T_{e j}\right)+a_{1 j}^{2}=0-T_{e j}+a_{1 j}^{2}=0, \\
& h_{2 j}\left(T_{e j}, b_{1 j}\right)=g_{2 j}\left(T_{e j}\right)+b_{1 j}^{2}=T_{e j}-b+b_{1 j}^{2}=0,
\end{aligned}
$$

where $a_{1 j}$ and $b_{1 j}$ are the slack variables, respectively. 
So the Lagrange equation is [30]

$$
\begin{aligned}
F\left(T_{e j}, \varepsilon, a_{1 j}, b_{1 j}, \mu_{1 j}, \mu_{2 j}\right) & =f\left(T_{e j}\right)+\varepsilon h\left(T_{e j}\right)+\mu_{1 j} h_{1 j}\left(T_{e j}, a_{1 j}\right)+\mu_{2 j} h_{2 j}\left(T_{e j}, b_{1 j}\right) \\
& =\sum_{j=1}^{4} \frac{1}{2} a_{j} T_{e j}^{2}+\varepsilon\left(\sum_{j=1}^{4} T_{e j}-T_{e}^{*}\right)+\mu_{1 j}\left(-T_{e j}+a_{1 j}^{2}\right)+\mu_{2 j}\left(T_{e j}-b+b_{1 j}^{2}\right),
\end{aligned}
$$

where $\varepsilon$ is the Lagrange multiplier and $\mu_{1 j} \geq 0$ and $\mu_{2 j} \geq 0$ are the KKT multipliers.

After the derivation of partial derivatives $T_{e 1}, T_{e 2}, T_{e 3}, T_{e 4}, \varepsilon, a_{1 j}, b_{1 j}, \mu_{1 j}$, and $\mu_{2 j}$ in (7),

$$
\left\{\begin{array}{l}
\frac{\partial F}{\partial T_{e j}}=a_{j} T_{e j}+\varepsilon-\mu_{1 j}+\mu_{2 j}, \\
\frac{\partial F}{\partial \varepsilon}=\sum_{j=1}^{4} T_{e j}-T_{e}^{*}, \\
\frac{\partial F}{\partial a_{1 j}}=2 \mu_{1 j} a_{1 j}, \\
\frac{\partial F}{\partial b_{1 j}}=2 \mu_{2 j} b_{1 j}, \\
\frac{\partial F}{\partial \mu_{1 j}}=h_{1 j}\left(T_{e j}, a_{1 j}\right)=g_{1 j}\left(T_{e j}\right)+a_{1 j}^{2} \\
\frac{\partial F}{\partial \mu_{2 j}}=h_{2 j}\left(T_{e j}, b_{1 j}\right)=g_{2 j}\left(T_{e j}\right)+b_{1 j}^{2}
\end{array}\right.
$$

The KKT condition indicates that the energy function must be satisfied when it reaches the minimum, and the derivations of partial derivatives in (8) are 0 . Setting $\mu_{1 j}=\mu_{2 j} \geq 0$, that is,

$$
\left\{\begin{array}{l}
a_{1} T_{e 1}=a_{2} T_{e 2}=a_{3} T_{e 3}=a_{4} T_{e 4} \\
\sum_{j=1}^{4} T_{e j}=T_{e}^{*} \\
\mu_{1 j} a_{1 j}=\mu_{2 j} b_{1 j}=0 \\
g_{1 j}\left(T_{e j}\right)+a_{1 j}^{2}=0 \\
g_{2 j}\left(T_{e j}\right)+b_{1 j}^{2}=0
\end{array}\right.
$$

where the detailed explanation can be found in [31]; thus,

$$
a_{1} T_{e 1}=a_{2} T_{e 2}=a_{3} T_{e 3}=a_{4} T_{e 4} .
$$

Also, with the $p$ percentage traction torque loss of the fourth motor, each traction torque matrix $T=\left[T_{e 1}, T_{e 2}\right.$, $\left.T_{e 3}, T_{e 4}\right]^{T}$ in (4) becomes $T_{c}=\left[T_{e 1}, T_{e 2}, T_{e 3},(1-p) T_{e 4}\right]^{T}$, and $T_{c}$ is the changed traction torque matrix. At the same time, by changing the initial weight matrix $P$, let it becomes the changed

weight

matrix $P_{c}=\operatorname{diag}\left((1-p) a_{1},(1-p) a_{2},(1-p) a_{3}, a_{4}\right)$. Finally, (10) can still be established.

Therefore, when the traction torque changes, the total traction is maintained at a constant under the constraint condition and the energy function value can be minimized by changing the weight coefficient matrix $P$.

\section{Sliding Mode Tracking Controller for Independent Motors}

In a variable structure control system, the motion of the system can be divided into two stages. The first stage is the stage of reaching motion, that is, the approaching process in the sliding mode control in which the reaching condition ensures that the system state trajectory reaches the switching surface from any initial state within a finite time. The second stage is that the error converges to zero under the influence of control law. This control method makes the system state slide along the sliding surface by switching the control quantity, thereby making the system invariant when subjected to parameter perturbation and external disturbance; thus, it has the advantages of rapid dynamic response and strong robustness in multimotors system coordination control, aircraft motion control, and other fields [32]. For this purpose, a variable structure control was introduced for improving the dynamic response performance of each independent motor.

The tracking error between the output torque of motor $j$ and the setting command is to be $e_{j}$; that is,

$$
e_{j}=T_{e j}-T_{e j}^{*} \text {. }
$$

After the derivation of (11),

$$
\dot{e}=\dot{T}_{e j}-\dot{T}_{e j}^{*}
$$

Formula (3) is substituted into (12); that is,

$$
\begin{aligned}
\dot{e}= & -J_{e q j} a_{0 j} x_{1 j}+\left(b_{e q j}-J_{e q j} a_{1 j}\right) x_{2 j} \\
& +J_{e q j} b_{j} u_{j}+d_{3 j}-\dot{T}_{e j}^{*} .
\end{aligned}
$$

Integral sliding surface is defined as

$$
s=e_{j}(t)+c \int_{0}^{t} \operatorname{sgn}\left(e_{j}(\tau)\right) \mathrm{d} \tau,
$$

where $c$ is the positive constant to be designed and $\operatorname{sgn}(\cdot)$ is a sign function.

The integral sliding mode control law is designed based on the nonlinear system shown in (3) and the 
integral sliding surface shown in (14), which can be expressed as follows:

$$
\begin{aligned}
u_{j}= & -\frac{1}{J_{e q j} b_{j}}\left[-J_{e q j} a_{0 j} x_{1 j}+\left(b_{e q j}-J_{e q j} a_{1 j}\right) x_{2 j}-\dot{T}_{e j}^{*}\right. \\
& \left.+\operatorname{csgn}\left(e_{j}\right)-\eta_{j} \operatorname{sgn}(s)\right],
\end{aligned}
$$

where $j=1,2,3$, and 4 and $\eta_{j}$ is the positive constant to be designed.

The controller contains unknown interference $d_{j}$. Thus, the influence of $d_{j}$ can be offset by selecting a sufficiently large switching gain $\eta_{j}$ to converge the error $e_{j}$ to 0 in a finite time.
Proof. Lyapunov function is defined as

$$
V=\frac{1}{2} s^{2} .
$$

After the derivation of $s$,

$$
\begin{aligned}
\dot{s}= & \dot{e}_{j}+c \operatorname{sgn}\left(e_{j}\right)=-J_{e q j} a_{0 j} x_{1 j}+\left(b_{e q j}-J_{e q j} a_{1 j}\right) x_{2 j} \\
& +J_{e q j} b_{j} u_{j}+d_{3 j}-\dot{T}_{e j}^{*}+c \operatorname{sgn}\left(e_{j}\right) .
\end{aligned}
$$

Then,

$$
\begin{aligned}
\dot{V}= & s \dot{s}=s\left(-J_{e q j} a_{0 j} x_{1 j}+\left(b_{e q j}-J_{e q j} a_{1 j}\right) x_{2 j}+J_{e q j} b_{j} u_{j}+d_{3 j}-\dot{T}_{e j}^{*}+c \operatorname{sgn}\left(e_{j}\right)\right) \\
= & s\left(-J_{e q j} a_{0 j} x_{1 j}+\left(b_{e q j}-J_{e q j} a_{1 j}\right) x_{2 j}+J_{e q j} b_{j}\left\{-\frac{1}{J_{e q j} b_{j}}\left[-J_{e q j} a_{0 j} x_{1 j}+\left(b_{e q j}-J_{e q j} a_{1 j}\right) x_{2 j}-\dot{T}_{e j}^{*}+c \operatorname{sgn}\left(e_{j}\right)+\eta_{j} \operatorname{sgn}(s)\right]\right\}\right. \\
& \left.+d_{3 j}-\dot{T}_{e j}^{*}+\operatorname{csgn}\left(e_{j}\right)\right)=s \cdot\left(-\eta_{j} \cdot \operatorname{sgn}(s)+d_{3 j}\right)=-\eta_{j}|s|+s d_{3 j} \leq-\eta_{j}|s|+|s|\left|d_{3 j}\right|=|s|\left(\left|d_{3 j}\right|-\eta_{j}\right) .
\end{aligned}
$$

Set $\eta_{j}$; if $\eta_{j} \geq\left|d_{3 j}\right|$ is met, then

$$
|s|\left(\left|d_{3 j}\right|-\eta_{j}\right) \leq 0 .
$$

Accordingly,

$$
\dot{V} \leq 0 \text {. }
$$

Meanwhile, the error $e_{j}$ gradually converges to 0 from the initial state, such that the output torque of each independent motor can be tracked to the reference state $T_{e j}^{*}$ in a limited time.

This completes the proof.

\section{Simulation Analysis}

The traction system that consists of four motors was taken as the object, and the parameters are shown in Table 1.

The initial weight coefficient in (4) is selected as $a_{j}=\operatorname{diag}[0.7,0.6,0.5,0.4]$. The parameter to be designed on the switching function in (15) is selected as $c=2$, and the parameter to be designed in (15) is selected as $\eta_{j}=[45,35,25,30]^{T}$.

In order to reflect three processes including the acceleration, uniform speed, and braking, the corresponding reference command is set as

$$
\begin{aligned}
& t<10, \quad \text { acceleration, } \\
& 10 \leq t \leq 20, \quad \text { uniform speed, } \\
& 20<t \leq 30, \quad \text { braking. }
\end{aligned}
$$

Four types of noise signals with high frequency, mutation, slow-varying, and uniformity are superimposed on the four traction motors, respectively, at $12 \mathrm{~s}$ to verify the dynamic response performance and noise interference suppression performance of the proposed method (see Figure 2).

The simulation is conducted on the system, that is, at $15 \mathrm{~s}, 100 \%$ loss and $30 \%$ loss of traction torque for a motor.

5.1. 100\% Traction Torque Loss of a Motor. When the fourth motor loses $100 \%$ traction torque, the changing curve of the total traction torque and each motor traction torque are shown in Figure 3. The solid black line indicates the total given traction torque $T_{e}^{*}$, the red dotted line indicates the sum of the traction torque of the four motors Te_total, and the lower coloured short line indicates each traction torque $T e_{j}(j=1,2,3,4)$, respectively.

Figure 3 shows that, in the system acceleration phase of $0-10 \mathrm{~s}$, the proportional coefficient assigned to the four motors is unchanged, and the sum of the output traction torques of the four motors is consistent with the total traction torque required. At $12 \mathrm{~s}$, the total traction torque fluctuates with the maximum error of $0.12 \%$. At $15 \mathrm{~s}$, the traction torque output of the fourth motor is 0 due to the loss of traction, and the required traction torque is distributed to 
TABle 1: Parameters of each motor.

\begin{tabular}{lcccc}
\hline Symbol & Motor 1 & Motor 2 & Motor 3 & Motor 4 \\
\hline Motor resistance $R$ & 2.5 & 2.2 & 2.3 & 2.4 \\
Motor inductance $L$ & 0.612 & 0.55 & 0.6 & 0.58 \\
Equivalent viscous friction coefficient $b_{e q}$ & 0.08 & 0.06 & 2.35 & 0.075 \\
Equivalent motor moment of inertia $J_{e q}$ & 2.4 & 2.3 & 81.8 & 8.15 \\
Motor torque coefficient $k_{m}$ & 82.2 & 81.5 & 82.3 & 82.31 \\
Back electromotive force constant $k_{e}$ & 82.32 & 7.888 & 7.9 & 7.95 \\
Gearbox transmission ratio $k_{t}$ & 8 & & & 82.3 \\
\hline
\end{tabular}

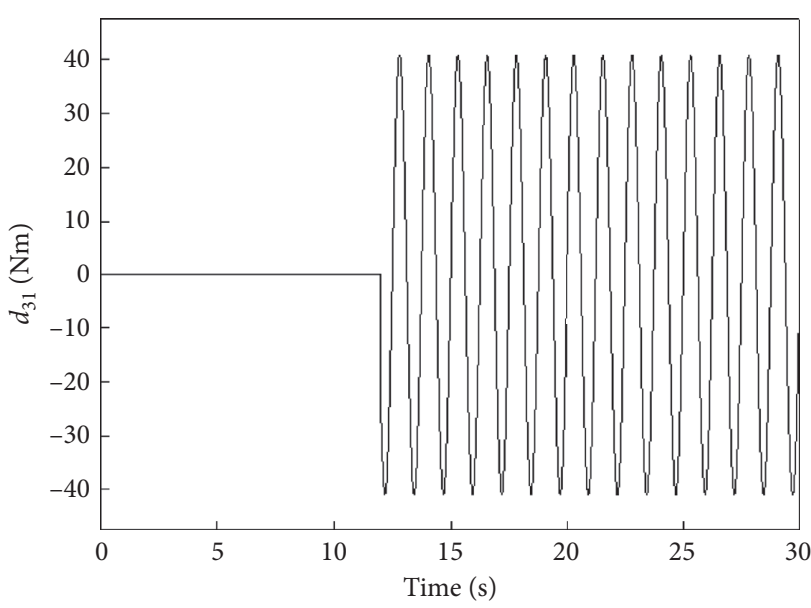

$-d_{31}$

(a)

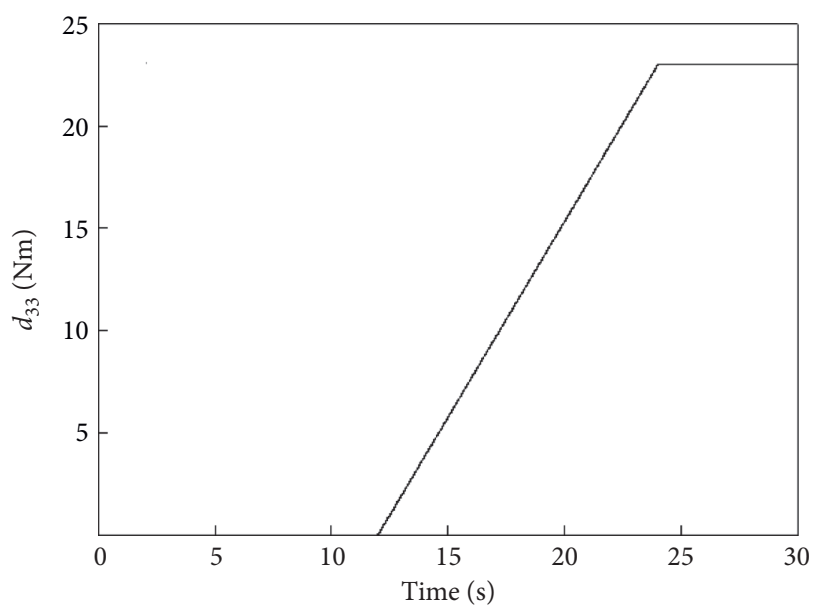

$-d_{33}$

(c)

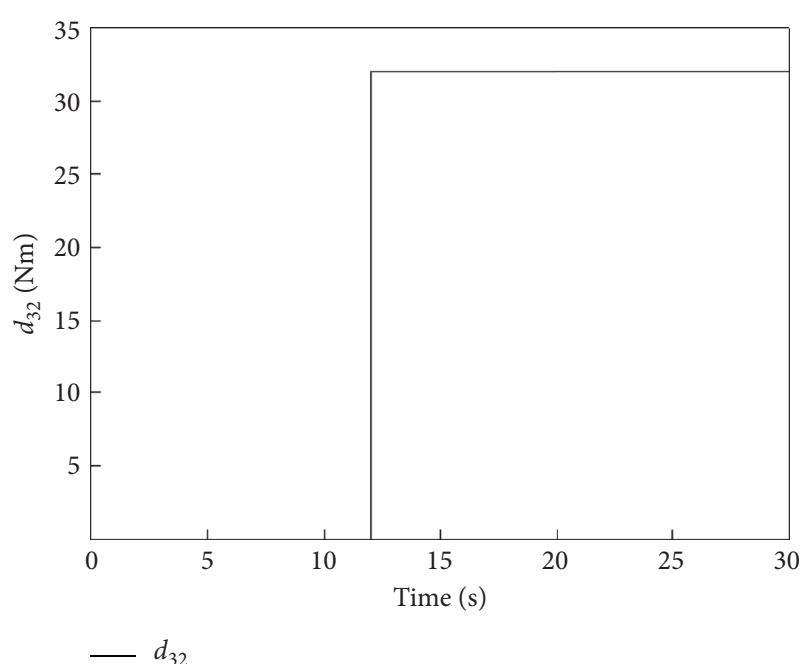

(b)

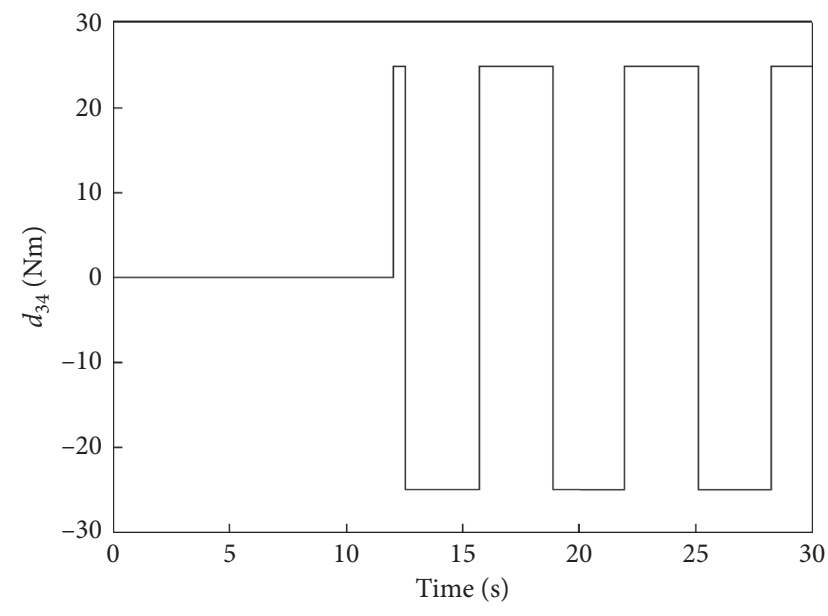

(d)

FIGURE 2: Four types of noise signal superimposed on four motors. (a) Interference signal 1. (b) Interference signal 2. (c) Interference signal 3. (d) Interference signal 4.

the other three motors. The maximum tracking error during the tracking of total quantity is $6 \%$, and the tracking time is $0.3 \mathrm{~s}$. Thus, the system can achieve total traction torque when a motor loses $100 \%$ traction torque.

To reflect the tracking performance of each independent motor more clearly, the traction torque change curve of the four motors in Figure 3 is shown in Figure 4. The solid black line indicates the assigned command signal, and the red dotted line indicates the output torque of each independent motor.

Figure 4 shows that, under the given noise signal interference at $12 \mathrm{~s}$, each motor has different degrees of 


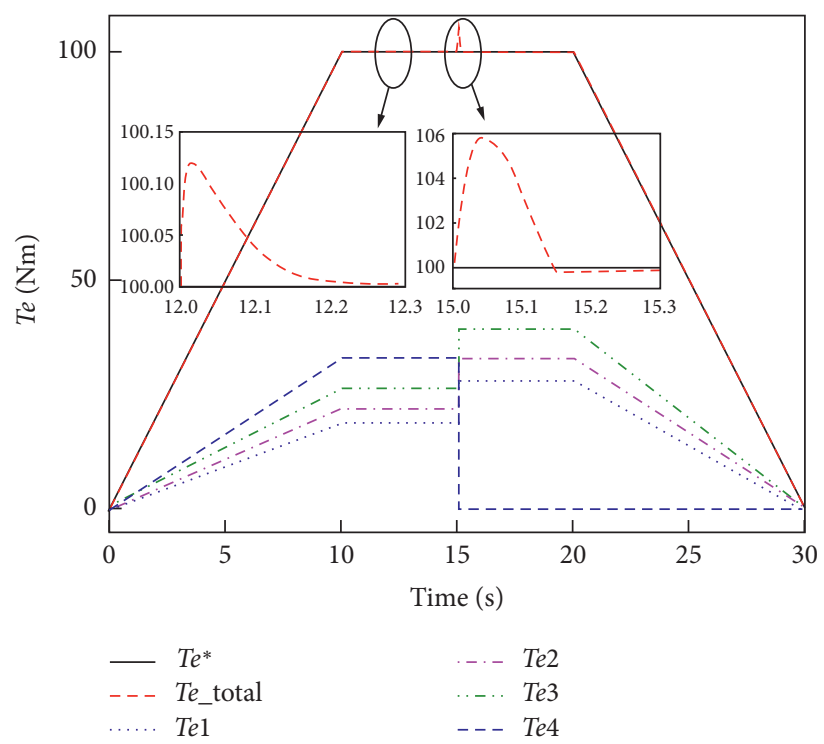

FIgURE 3: The changing curve of the total traction torque and each motor traction torque output when the fourth motor loses $100 \%$ traction torque.

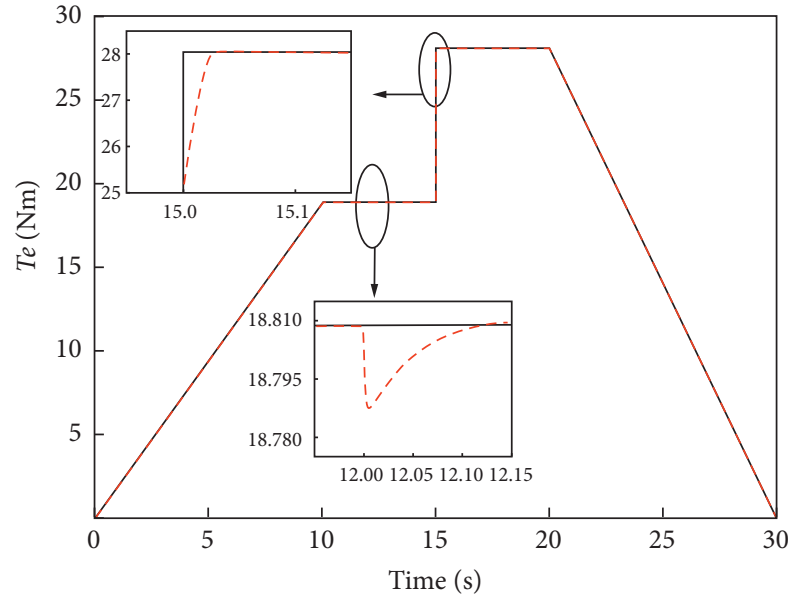

$-\mathrm{Te}^{*}$

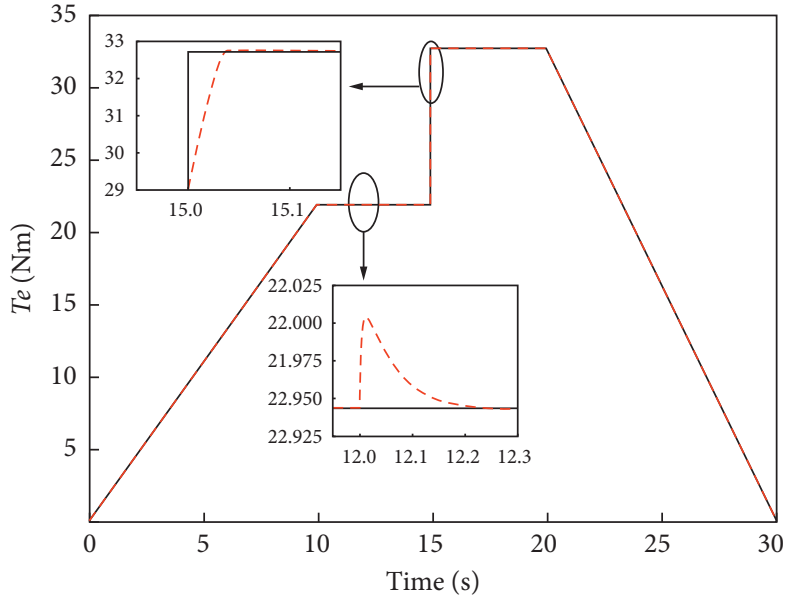

$-\mathrm{Te} 2^{*}$

(a)

(b)

Figure 4: Continued. 


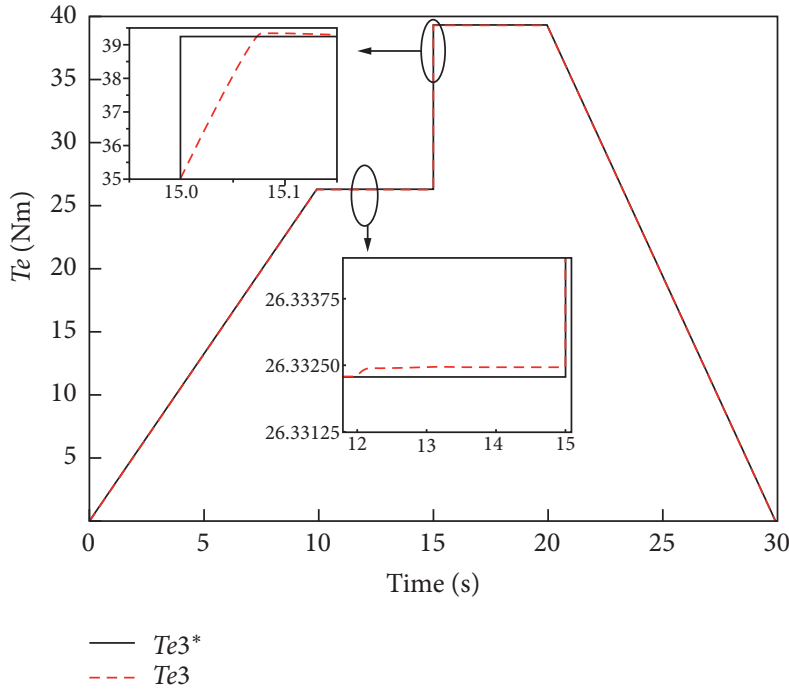

(c)

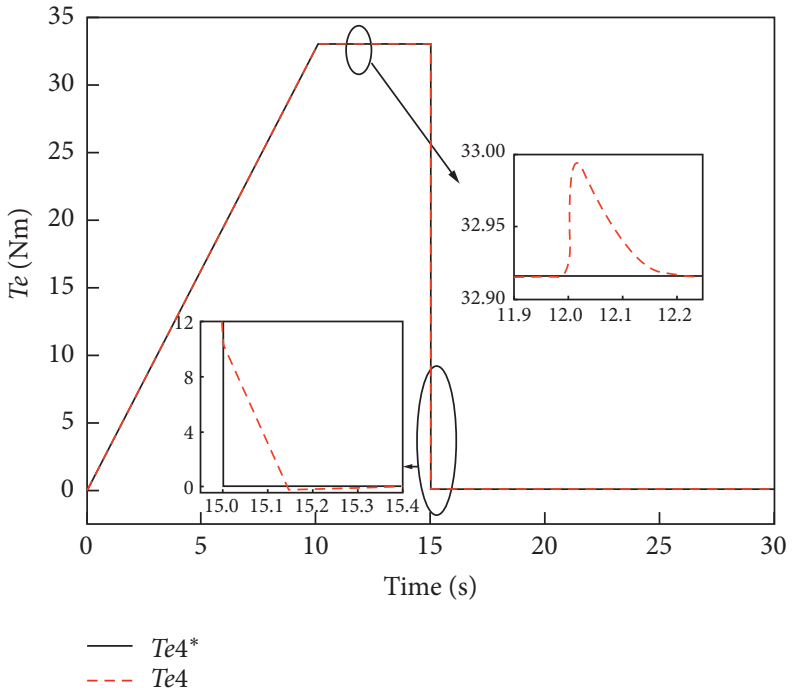

(d)

Figure 4: Output torque change curve of the four motors when the fourth motor loses $100 \%$ traction. (a) Output torque change curve of motor 1. (b) Output torque change curve of motor 2. (c) Output torque change curve of motor 3. (d) Output torque change curve of motor 4.

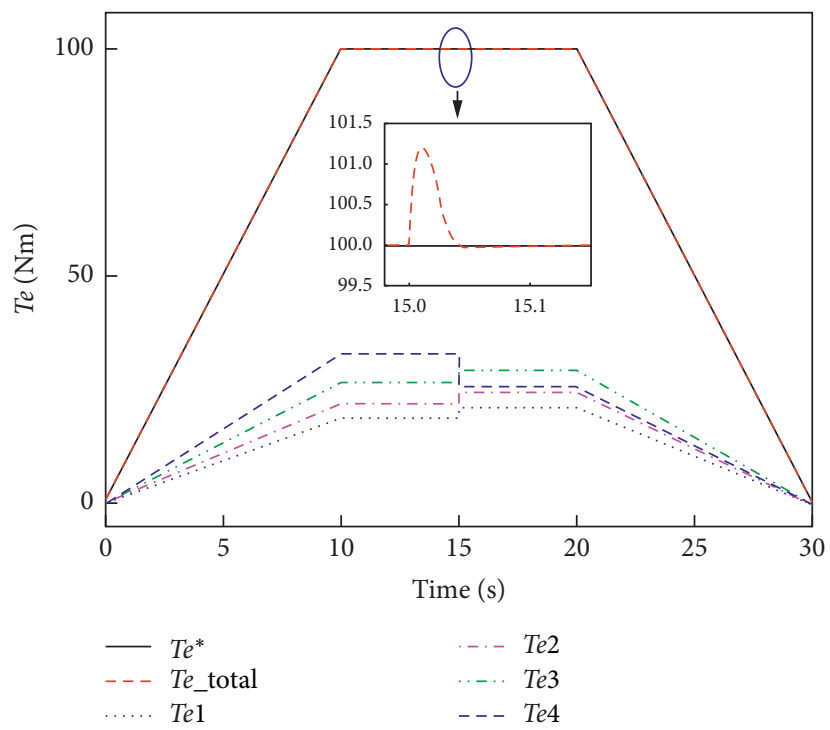

FIgURE 5: The changing curve of the total traction torque and each motor traction torque output when the fourth motor loses $30 \%$ traction torque.

fluctuation, and the fluctuation quantities are $0.03 \%, 0.05 \%$, $0.01 \%$, and $0.1 \%$ with tracking time of $0.15,0.3,2.8$, and $0.2 \mathrm{~s}$, respectively. At $15 \mathrm{~s}$, the traction torques of the three remaining motors show different degrees of overshoot during the transition to the torque required for redistribution due to the complete loss of traction of the fourth motor, and the overshoot is $10.7 \%, 11.6 \%$, and $10.6 \%$ with stability time of $0.04 \mathrm{~s}, 0.15 \mathrm{~s}$, and $0.15 \mathrm{~s}$, respectively. Notably, after the fourth motor loses traction torque, the torque does not immediately become 0 and the fluctuation existed due to the influence of sliding mode. Thus, when noise exists, the motor torque output has high tracking performance using the proposed method.

5.2. 30\% Traction Torque Loss of a Motor. When the fourth motor loses $30 \%$ of traction torque, the changing curve of the total traction torque and the each motor traction torque is shown in Figure 5. The solid black line indicates the given total traction $T_{e}^{*}$, the red dotted line indicates the sum of the traction torque of the four motors Te_total, and the lower 


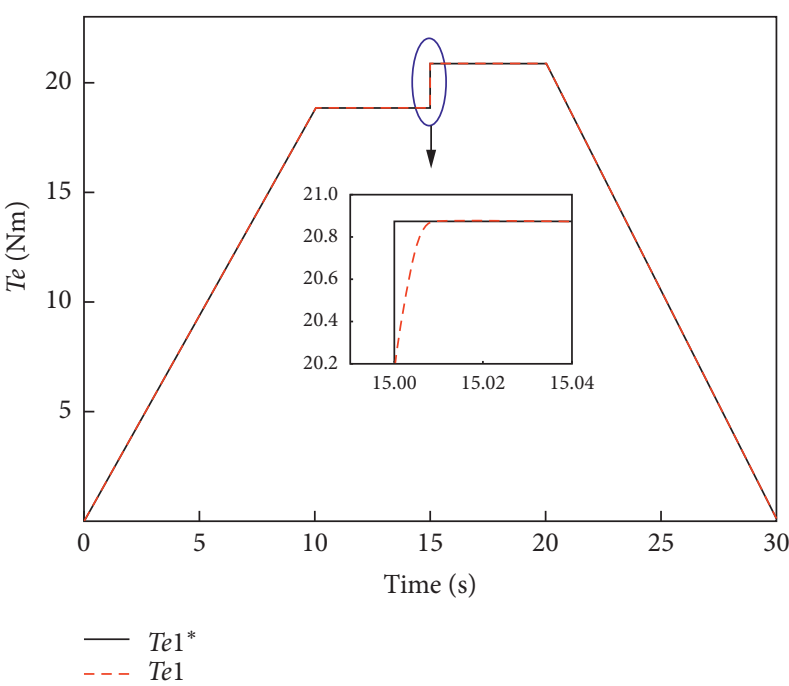

(a)

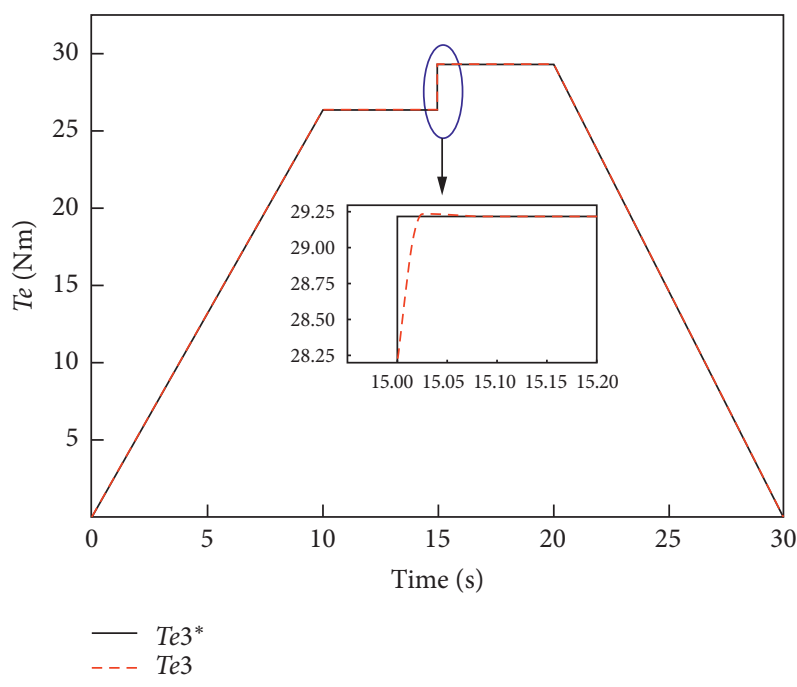

(c)

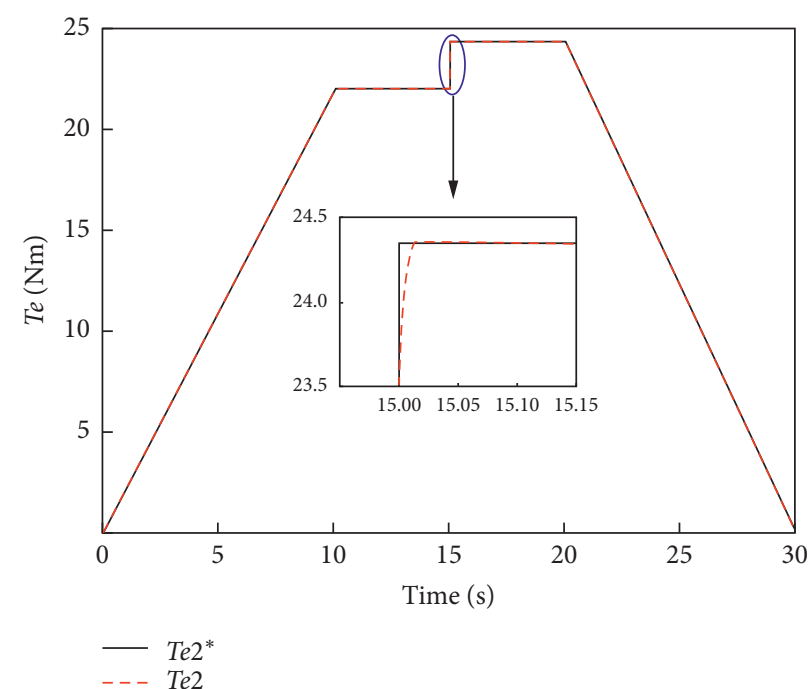

(b)

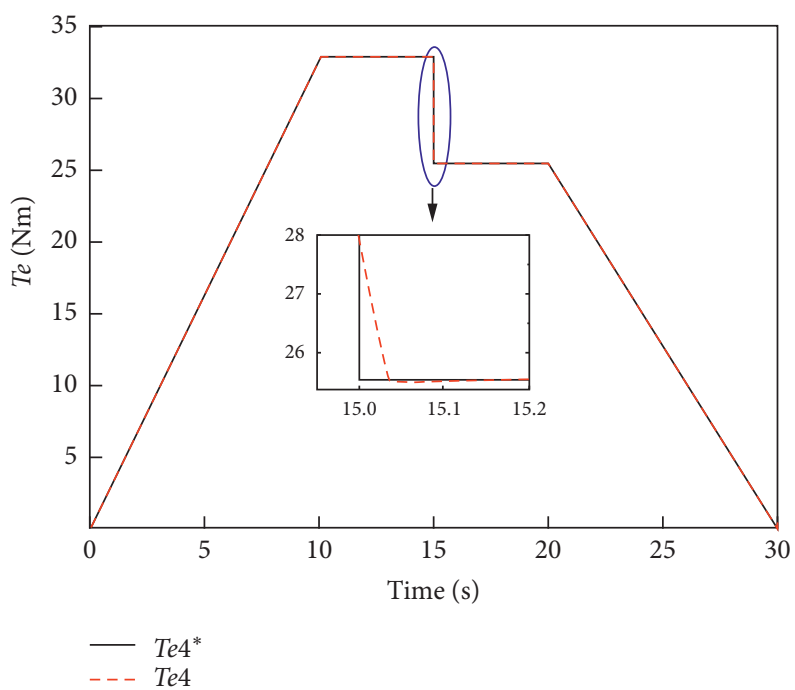

(d)

Figure 6: Output torque change curve of the four motors when the fourth motor loses $30 \%$ traction. (a) Output torque change curve of motor 1. (b) Output torque change curve of motor 2. (c) Output torque change curve of motor 3. (d) Output torque change curve of motor 4.

coloured short line indicates each traction torque $T e_{j}(j=1,2,3,4)$.

Figure 5 shows that, at $15 \mathrm{~s}$, the maximum tracking error during the tracking process of total traction torque is $1.3 \%$ due to the $30 \%$ traction loss of the fourth motor, and the tracking time is $0.1 \mathrm{~s}$. Thus, the system can achieve total traction torque when a motor loses $30 \%$ traction torque.

The torque change curve of the four motors in Figure 5 is shown in Figure 6 . The solid black line indicates the assigned command signal, and the red dotted line indicates the output torque of each independent motor.

Figure 6 shows that when the fourth motor loses 30\% traction torque at $15 \mathrm{~s}$, the output torque of the fourth motor is set to $70 \%$ of the original output torque, and the required traction torque is mainly compensated by the remaining motors. In the process of output torque coordination, the output torque fluctuations of the four motors are $3.21 \%, 3.24 \%$,
$3.29 \%$, and $9.43 \%$ with tracking time of $0.04,0.15,0.2$, and $0.2 \mathrm{~s}$, respectively. Thus, when the motor loses part of the traction, the system still has preferable dynamic response performance.

\section{Conclusions}

The consistent total traction torque in the operation of electric locomotives is the premise of safe and stable operation of locomotives. A multimotors coordination control method for consistent total traction torques is proposed to ensure that the sum of multimotors output traction torque of electric locomotives is consistent with the total traction torque required. The main innovations of this study are as follows: firstly, a multimotors coordination control framework is constructed for consistent total traction torque. Secondly, a multimotors allocation control strategy, which provides an idea for optimising the multimotors dynamic 
adjustment process, is proposed based on minimisation of energy function. Thirdly, a traction torque control strategy based on sliding mode variable structure is constructed for each independent motor. Finally, the simulation environments under two different conditions are constructed to verify the proposed method. The results show that the proposed method shows good performance in terms of consistency of total traction torque, as well as dynamic response performance of an independent motor.

\section{Data Availability}

The survey data used to support the findings of this study are available from the corresponding author upon request.

\section{Disclosure}

The views expressed here are solely the responsibility of the authors.

\section{Conflicts of Interest}

The authors declare that they have no conflicts of interest.

\section{Acknowledgments}

This work was supported in part by the Natural Science Foundation of China (Grant no. 61773159), Excellent Youth Research Project of Education Department of Hunan Province (18B303), and Hunan Provincial Natural Science Foundation of China (Grant no. 2018JJ2100).

\section{References}

[1] Y. Liu, B. Zou, A. Ni, L. Gao, and C. Zhang, "Wheel/rail adhesion state identification of heavy-haul locomotive based on particle swarm optimization and kernel extreme learning machine," Journal of Advanced Transportation, vol. 2020, Article ID 8136939, 6 pages, 2020.

[2] A. A. Zarifian and P. G. Kolpahchyan, "Computer modeling of electric locomotive as controlled electromechanical system," Multibody System Dynamics, vol. 22, no. 4, pp. 425-436, 2009.

[3] J. U. Duncombe, "Infrared navigation-part I: an assessment of feasibility," IEEE Transactions on Electron Devices, vol. 11, no. 1, pp. 34-39, 1959.

[4] A. Hoffrichter, P. Fisher, J. Tutcher, S. Hillmansen, and C. Roberts, "Performance evaluation of the hydrogen-powered prototype locomotive "Hydrogen Pioneer"," Journal of Power Sources, vol. 250, pp. 120-127, 2014.

[5] K. Song, G. Konstantinou, W. Mingli, and P. Acuna, "Windowed SHE-PWM of interleaved four-quadrant converters for resonance suppression in traction power supply systems," IEEE Transactions on Power Electronics, vol. 32, no. 10, pp. 7870-7881, 2017.

[6] C. Zhang, Z. Lin, J. Liu, and J. He, "Consensus-based totalamount cooperative tracking control for multi-motor locomotive traction system," Journal of the Franklin Institute, vol. 356, no. 2, pp. 819-834, 2019.

[7] W.-C. Cai, D.-Y. Li, and Y.-D. Song, "A novel approach for active adhesion control of high-speed trains under antiskid constraints," IEEE Transactions on Intelligent Transportation Systems, vol. 16, no. 6, pp. 3213-3222, 2015.

[8] W. Zhang, J. Chen, X. Wu, and X. Jin, "Wheel/rail adhesion and analysis by using full scale roller rig," Wear, vol. 253, no. 1-2, pp. 82-88, 2002.

[9] R. Galas, M. Omasta, I. Krupka, and M. Hartl, "Laboratory investigation of ability of oil-based friction modifiers to control adhesion at wheel-rail interface," Wear, vol. 368-369, pp. 230-238, 2016.

[10] J. H. Feng, "Research on the permanent magnet synchronous motor drive system for high-speed EMUs," Journal of Electric Drive for Locomotives, no. 4, pp. 1-5, 2016, in Chinese.

[11] N. R. Abjadi, J. Askari, J. Soltani, and G. R. Arab Markadeh, "Nonlinear sliding-mode control of a multi-motor webwinding system without tension sensor," IET Control Theory \& Applications, vol. 3, no. 4, pp. 419-427, 2009.

[12] C. Zhang, M. Niu, J. He, and K. Zhao, "Robust synchronous control of multi-motor integrated with artificial potential field and sliding mode variable structure," IEEE Access, vol. 5, pp. 197-207, 2017.

[13] M.-T. Yan, M.-H. Lee, and P.-L. Yen, "Theory and application of a combined self-tuning adaptive control and cross-coupling control in a retrofit milling machine," Mechatronics, vol. 15, no. 2, pp. 193-211, 2005.

[14] H. Hou, X. Nian, H. Xiong, Z. Wang, and Z. Peng, "Robust decentralized coordinated control of a multimotor webwinding system," IEEE Transactions on Control Systems Technology, vol. 24, no. 4, pp. 1495-1503, 2016.

[15] J. Liao, Z. Chen, and B. Yao, "Performance-oriented coordinated adaptive robust control for four-wheel independently driven skid steer mobile robot," IEEE Access, vol. 5, pp. 19048-19057, 2017.

[16] C. Zhang, H. Wu, J. He, and C. Xu, "Consensus tracking for multi-motor system via observer based variable structure approach," Journal of the Franklin Institute, vol. 352, no. 8, pp. 3366-3377, 2015.

[17] T. Shi, H. Liu, Q. Geng, and C. Xia, "Improved relative coupling control structure for multi-motor speed synchronous driving system," IET Electric Power Applications, vol. 10, no. 6, pp. 451-457, 2016.

[18] C. Zhang, Z. Lin, S. X. Yang, and J. He, "Total-Amount synchronous control based on terminal sliding-mode control," IEEE Access, vol. 5, pp. 5436-5444, 2017.

[19] Z. Zhang, Y. Zhao, W. Qiao, and L. Qu, "A space-vectormodulated sensorless direct-torque control for direct-drive PMSG wind turbines," IEEE Transactions on Industry Applications, vol. 50, no. 4, pp. 2231-2341, 2014.

[20] C. Zhang, H. Liao, X. Li, J. Sun, and J. He, "Fault reconstruction based on sliding mode observer for current sensors of PMSM," Journal of Sensors, vol. 2016, Article ID 9307560, 9 pages, 2016.

[21] W. Xu and R. D. Lorenz, "Dynamic loss minimization using improved deadbeat-direct torque and flux control for interior permanent-magnet synchronous machines," IEEE Transactions on Industry Applications, vol. 50, no. 2, pp. 1053-1065, 2014.

[22] Y. S. Choi, H. C. Han, and J. W. Jung, "Feedback linearization direct torque control with reduced torque and flux ripples for IPMSM drives," IEEE Transactions on Power Electronics, vol. 31, no. 5, pp. 3728-3737, 2015.

[23] F. A. Rahimi, M. G. Lauby, J. N. Wrubel, and K. L. Lee, "Evaluation of the transient energy function method for online dynamic security analysis," IEEE Transactions on Power Systems, vol. 8, no. 2, pp. 497-507, 2002. 
[24] M. Levitt, M. Hirshberg, R. Sharon, and V. Daggett, "Potential energy function and parameters for simulations of the molecular dynamics of proteins and nucleic acids in solution," Computer Physics Communications, vol. 91, no. 1-3, pp. 215-231, 1995.

[25] R. Yousefian, A. Sahami, and S. Kamalasadan, "Hybrid transient energy function-based real-time optimal wide-area damping controller," IEEE Transactions on Industry Applications, vol. 53, no. 2, pp. 1506-1516, 2017.

[26] H. Shao, H. Jiang, H. Zhang, and T. Liang, "Electric locomotive bearing fault diagnosis using a novel convolutional deep belief network," IEEE Transactions on Industrial Electronics, vol. 65, no. 3, pp. 2727-2736, 2017.

[27] C. Mayet, J. Pouget, A. Bouscayrol, and W. Lhomme, "Influence of an energy storage system on the energy consumption of a diesel-electric locomotive," IEEE Transactions on Vehicular Technology, vol. 63, no. 3, pp. 1032-1040, 2014.

[28] F. Beltran-Carbajal, A. Valderrabano-Gonzalez, J. C. RosasCaro, and A. Favela-Contreras, "An asymptotic differentiation approach of signals in velocity tracking control of DC motors," Electric Power Systems Research, vol. 122, pp. 218223, 2015 .

[29] Y. D. Song, J. N. Anderson, and H. Y. Lai, "Robust control of multi-robot systems: the generalized energy accumulation approach," International Journal of Robust \& Nonlinear Control, vol. 4, no. 5, pp. 673-696, 2010.

[30] C. Mahapatra, Z. Sheng, P. Kamalinejad, V. C. M. Leung, and S. Mirabbasi, "Optimal power control in green wireless sensor networks with wireless energy harvesting, wake-up radio and transmission control," IEEE Access, vol. 5, pp. 501-518, 2016.

[31] M. Li, "Generalized Lagrange multiplier method and KKT conditions with an application to distributed optimization," IEEE Transactions on Circuits and Systems II: Express Briefs, vol. 66, no. 2, pp. 252-256, 2019.

[32] S. Laghrouche, F. Plestan, and A. Glumineau, "Higher order sliding mode control based on integral sliding mode," Automatica, vol. 43, no. 3, pp. 531-537, 2007. 